\title{
Yeast metabolic products, yeast antigens and yeasts as possible triggers for irritable bowel syndrome Heiko Santelmanna and John McLaren Howardb
}

\author{
Eur J Gastroenterol Hepatol 2005; 17(1): 21-26 \\ Keywords: candida, allergy, hypersensitivity, IBS, gut fermentation \\ aHolistisk Senter, Oslo, Norway, and ıBiolab Medical Unit, London, UK. \\ Correspondence to Heiko Santelmann, Holmenveien 1, N-0374 Oslo, Norway. \\ Tel: +47-22139510; fax: +47-22139513; e-mail: drheiko@online.no; www.candidaallergy.com
}

\begin{abstract}
Many patients with irritable bowel syndrome (IBS) are disillusioned by the lack of efficacy of treatments and suffer from numerous symptoms not covered by the Rome criteria for IBS, as the current empirical treatment regimes fail to address these persistent debilitating 'IBS associated symptoms'. These symptoms are similar to other symptom complexes like chronic fatigue and the so called 'candida syndrome', and many seek help from alternative medicine. The possible role of Candida and yeasts in non-immune compromised individuals is disputed and is the subject of this review.

Even if the involvement of yeasts in the aetiology of IBS still remains unclear, there is increasing evidence for yeasts being able to cause IBS-symptoms in sensitized patients via Candida products, antigens and cross-antigens. But more research is needed before antifungal treatment can be recommended as a first line treatment for IBS.
\end{abstract}

\section{Introduction}

Most physicians and gastroenterologists acknowledge that the medical treatment for irritable bowel syndrome (IBS) is empirical, not necessarily based on pathophysiological findings and substantially lacking in efficacy. Thus, increasingly disillusioned, patients are left with persistent and significant symptoms which may interfere with their ability to cope with certain stressful life events as reflected by absence from work, marital disharmony and increased use of health resources.

The definition of IBS has recently been placed on firmer grounds with the symptom based Rome criteria. However, it seems clear that patients with IBS have many symptoms that these criteria do not cover. These include bloating, abdominal distension, food intolerances that do not appear to have a rational biochemical explanation, chemical sensitivities, reduced tolerance of alcohol, flatulence and bulimia. There are also extraintestinal symptoms such as profound tiredness, depression, anxiety, lack of self confidence, arthralgia, myalgia, pre-menstrual and perimenstrual tension, for example. Lack of interest in these symptoms may be due to our inability to treat them effectively. However, this leaves many patients with the perception of a lack of coordinated attention to their diverse symptoms and many seek help from alternative medicine.

It is becoming increasingly clear that the above IBS associated symptoms are similar to the symptom complexes of chronic fatigue syndrome and multiple chemical sensitivities. A common theme with these 'unexplained symptom complexes' is that the disability seems to be out of proportion to physical findings $[1,2]$. The place of these symptom based disorders within the nosology of clinical medicine is uncertain.

From the non-traditionalist gastroenterological point of view there have been evolving attempts to account for the 'unexplained clinical symptoms' associated with IBS. High carbohydrate intake in some of these patients prompted diagnostic labels such as germ-carbohydrate fermentation and intestinal carbohydrate dyspepsia [3] in the early 20th century, while later attempts include descriptions of the candidiasis hypersensitivity syndrome [4] gut fermentation syndrome [5] fungal type dysbiosis [6-9] and abnormal gut fermentation [10-12].

All these have in common a general tendency to describe IBS in terms of normal, or abnormal intestinal microbiological flora resulting in gut fermentation problems, yeast sensitivity and dysbiosis.

Others have observed that yeast overgrowth occurs in certain situations where there is abundance of carbohydrates [13-15] and it is suggested that the symptoms in patients with gut dysbiosis are worsened by exposure 
to yeasts and mould containing foods [7]. Much of this work is admittedly controversial. The idea of the candida syndrome or candida hypersensitivity, i.e., that intestinal colonization with Candida albicans is one cause of IBS symptoms or the 'unexplained symptom complex' of IBS, have received widespread publicity in the popular press. Patients are thus increasingly asking their health providers for information, clarification and treatment of yeast related issues. The possible role of Candida and yeasts in non-immune compromised individuals is disputed and is the subject of this review.

\section{Biology of Candida albicans}

The three major groups of fungi are moulds, yeasts and mushrooms. Candida are unicellular yeasts, somewhat larger than bacteria, that divide mostly asexually, can switch between a yeast- and a pseudohyphal or hyphal form and, like other yeasts, flourish in habitats where there is an abundance of sugar. Candida are normal human commensals, particularly in the mouth, skin, vagina and intestine. Candida can be cultured from faeces in up to $80 \%$ of healthy adults [16-18]. Candida numbers increase significantly following antibacterial therapy $[19,20]$, but the numbers seem to be unaffected by a refined carbohydrate diet [21]. It seems likely that intestinal Candida numbers are regulated in a similar way as intestinal bacteria $[22,23]$.

Candida albicans is the most common cause of candidasis in humans, but other species of Candida such as $C$. tropicalis, C. parapsilosis, C. krusei and C. glabrata may also be responsible. The pathogenesis of disease associated with Candida in humans is driven by host factors. Candida does not seem to produce specific mycotoxins [24] although some strains produce gliotoxin which may impair neutrophil function [25]. However, it is a polyantigenic organism containing up to 178 different antigens [26-28], which might explain the number of cross-reactions to yeasts (Malassezia, bread/brewers yeast) and moulds [28,29] and even human tissue [30].

It was shown recently that there is a potential cross reactivity with gluten because of several amino acid sequences that are highly homologous to alpha-gliadin and gamma-gliadin. Such a mechanism might lead to wheat intolerance with its accompanying symptoms and even trigger celiac disease in genetically susceptible people [31]. Furthermore, a placebo controlled crossover study revealed that dietary yeast may affect the activity of Crohn's disease [32]. Candida produces alcohol [10] and contains glycoproteins which have the potential to stimulate mast cells to release histamine and apparently prostaglandin (PGE2), inflammatory substances which could cause IBS-like symptoms [33-35]. Other circumstantial evidence support the theory of yeasts as trigger for the IBS: Secretory immunoglobulin A (SIgA) is front line in the defence of mucous membranes, especially in the intestine where it is active against infectious agents and certain antigens [36]. At least three different Candida species are able to produce proteases which can degrade IgA1, IgA2 and SIgA [37]. This protease activity can induce polyclonal B-cell response and inflammation. An infection of the intestinal mucosa with Candida might lead to an inactivating of SIgA and inflammation within subgroups of patients with IBS symptoms.

Candida is sensitive to a number of antifungal agents, such as nystatin, which is not absorbed from the gastrointestinal tract after oral administration. It destroys Candida by binding to sterols in the cell membrane, and thereby increasing permeability with loss of cellular contents.

\section{Epidemiology of intestinal Candida}

There are no satisfactory studies addressing intestinal colonization of Candida in IBS patients that support, or refute a causative effect. A recent review, however, concluded that there was a strong association between improvement of diarrhoeal symptoms in patients previously treated with antibacterials, who had a significant overgrowth of Candida in their stools and treatment with antifungal agents [38]. Renfro et al. [39] investigated 100 consecutive patients (using symptom based questionnaires) with chronic fatigue and compared those who believed that their 'unexplained symptom complex' and chronic fatigue was due to yeast to those who did not hold this belief. There were no specific features that supported the preconceived belief that Candida caused the fatigue. The authors attributed most of the symptoms to psychiatric factors.

A study from France suggested that the presence in the intestinal tract of yeasts (Candida albicans, Geotrichum candidum) caused IBS symptoms in atopic individuals [40]. However, antibodies to Candida are frequently seen in atopic and allergic individuals without IBS $[41,28]$. While some of these studies question the validity of a causative relationship between intestinal Candida and IBS, a lack of association might be due to methodological problems.

\section{Diagnostic tests}


Laboratory investigations into the possible role of yeast in polysymptomatic patients, especially those with IBS and chronic fatigue, have been problematic because there is no accepted 'gold standard' for distinguishing between a yeast sensitivity condition and a yeast overgrowth in the gut. Candida infection is well documented in vaginal and oral-oesophageal candidiasis. Biopsies and smears examined by microscopy, or cultures on yeastfriendly media are routinely used to identify the causative organism. However, establishing that yeast is pathogenically involved, is more problematic where the intestine is concerned

Stool tests for yeasts are commercially available, but they are used with limited and variable success. While it might be useful to examine stool or rectal smears by microscopy and/or culture for yeasts, these investigations usually do not distinguish between commensal yeasts and potentially pathogenic Candida [38,42].

Direct sampling of luminal contents of the small and/or large bowel in order to study yeast overgrowth has not been widely used because of the invasive nature of sample collection [7] and uncertainties regarding interpretation. Furthermore, Middleton et al. [43] did not reveal an association or a correlation between a group with IBS benefiting from antifungal treatment and microbiological findings of yeasts in stools.

Evidence for yeast as a causative factor for symptoms has rather been sought from indirect measurements that look for evidence of yeast metabolites in blood, breath or urine $[5,6,10,12,44-46]$, or that explore yeast allergy/sensitivity [12,44,47-53].

Analyses of studies that describe tests for Candida show that the analytical methods are sound, but in many cases the sensitivity and specificity of the methods has not been assessed extensively and the usefulness of some of the tests awaits confirmation. Some tests explore the possibility that intestinal yeasts metabolize an oral glucose load by measuring blood ethanol $1 \mathrm{~h}$ after ingestion of a test solution $[9,45,46]$. These tests have been further developed to include analyses of other alcohols that bacteria, rather than yeast, can produce $[6,10]$. The test attempts, and has the potential, to identify patients where yeast overgrowth is of sufficient magnitude to produce measurable metabolites in circulation and distinguishes this from bacterial overgrowth, or bacterial dysbiosis [6$8,11]$. Unfortunately, the glucose challenge test is not specific and cannot show that abnormal findings are due to Candida albicans or, for that matter, any other yeast.

A large number of allergy tests exist with extracts of Candida albicans used for prick or intradermal skin testing as well as for in vitro tests (RAST, ELISA). But the interpretation of test results is difficult, due to standardization problems with the extracts (different antigen quantity and quality from different Candida strains, grown on different growth media and antigens selected from different parts of the yeast), cross-reactivity between other yeasts and moulds and last not least different antibody responses (IgE, $\operatorname{IgA}, \operatorname{IgG}, \operatorname{IgM}$ ). Not surprisingly, many of these tests do not distinguish patients from normal controls [48-51]. Measurement of a combination of antigen, antibody and immune complexes in the serum may be most valuable in the diagnosis of fungus related disease [52-54]. Most promising are the new recombinant fungus allergens, which are clearly superior to fungal extracts for diagnostic purposes as deduced from the significant correlations obtained between skin test outcome and serology [29]. Another attempt to find patients belonging to a subgroup with IBS and polysymptomatic symptoms caused by Candida are questionnaires. Unfortunately, only very few of those have been validated. In an open, retrospective study, a widely used 70-item questionnaire [4] has been filled out by 380 patients who were evaluated after a threeweek treatment with the broad spectrum antifungal nystatin. The questionnaires from the responders were compared to those from the non-responders in a gradual statistical discrimination analysis. The statisticians concluded that by using only 7 questions ("Fungus-Related-Disease-Questionnaire-7", FRDQ-7, see Appendix 1) of the original 70item questionnaire, one could predict a positive response in $95 \%$ to the treatment used in this study [55].

\section{Treatment of 'candida syndrome'}

Proposed treatment of intestinal candidiasis involves dietary management and specific antifungal treatment. The dietary change usually involves a low carbohydrate diet. The rationale for this approach is that fungi thrive on carbohydrates. However, most complex carbohydrates are broken down into monosaccharides, which are absorbed in the proximal jejunum, although a small proportion of ingested sugar may survive as lactulose in lactasedeficient subjects.

There are several published Candida treatment trials. A group of volunteers was studied (mouth wash and stool yeast quantification and differentiation) before, during and after a high-sugar diet [21]. Refined carbohydrates had no significant effect on Candida numbers or subspecies in the mouth or intestine. 
In a small number of patients with 'Candida induced diarrhoea' (defined as the development of diarrhoea after a course of an antibiotic and which is associated with high faecal Candida levels) there appears to have been a $100 \%$ response following antifungal treatment [38]. In an open study of 25 patients (six of whom had received antibiotics) with prolonged diarrhoea (average 16 months) 18 (72\%) responded within a few days of nystatin treatment [56]. A response rate of over $90 \%$ of patients with flatulence treated with oral antifungal agents is difficult to interpret as there was no control group[57]. However, in a double-blind trial in women with recurrent vaginal candidiasis and the 'candida hypersensitivity syndrome' (multiple unexplained symptom complex, including gastrointestinal) oral and/or vaginal nystatin relieved the vaginal, but not the systemic symptoms [58]. Unfortunately, Dismukes and co-workers chose a crossover protocol, inappropriate for studying an antimicrobial agent [54].

A recent study again raises the possibility of antifungal treatment in selected polysymptomatic persons [55]. Patients from all parts of Norway were recruited (via one press advertisement) to take part in the study. Of 1620 patients who believed that their 'unexplained symptom complex' was due to yeast and who contacted the investigator, 954 were excluded according to predetermined criteria and of the remaining 666, 120 recorded high scores on the questionnaire FRDQ-7, indicating a probable 'fungus related complex'. These were randomly assigned to receive either nystatin or placebo in a double-blind, randomized placebo controlled, multi- centre trial with block design and diet as the block factor. Patients were asked to choose between continuation of their regular diet or a modified diet that was free from sugar, yeast and fungus antigens (see Appendix 2). The effect of treatment was monitored by a 45item symptom questionnaire. The difference between the nystatin groups (nystatin, nystatin + diet) and the placebo groups (placebo, placebo + diet) was highly significant $(\mathrm{P}<0,003)$. As was the difference between the groups following their regular diet or a diet free from sugar, yeast and fungus antigens. Unfortunately the diets have not been administered in a double-blind way.

The best improvement was seen in symptoms attributed to the central nervous system (fatigue, feeling of being drained, frequent mood swings, attacks of anxiety or crying, feeling spacey or unreal, poor memory, dizziness, insomnia, inability to make decisions and depression). Although most of the 120 volunteers had IBS-like symptoms, only 9 had actually been diagnosed with IBS. This small subgroup showed a significant improvement of symptoms (35\%) after taking nystatin, while the IBS-patients in the placebo groups improved with only $9 \%$.

\section{Conclusions}

Although the notion that intestinal Candida has an adverse effect on patients' health has been around for a long time, there has been little progress in the development of techniques that can diagnose the condition with any degree of sensitivity or specificity. The main diagnostic methods involve indirect evidence for the presence of yeasts. But even if the involvement of yeasts in the aetiology of IBS still remains unclear, there is increasing evidence for yeasts being able to cause IBS-symptoms in sensitized patients via Candida products, antigens and cross-antigens. Nevertheless, the main criticism of the idea of a fungus related syndrome in non-immune compromised or postantibiotic treated patients has been the lack of controlled treatment trials. In two recent controlled trials of antifungal treatment, one showed no significant effects on systemic symptoms, while the other reported a significant improvement of patients selected by a new questionnaire. This questionnaire (FRDQ-7) may be an effective tool to identify a subgroup with IBS related to yeasts and moulds. Additional improvement by a modified diet free from sugar, yeast and fungus antigens might even justify antifungal treatment.

Patients are increasingly questioning the efficacy of conventional treatment for the IBS, but more research is needed before antifungal treatment can be recommended as a first line treatment for IBS.

\section{References}

1 Aaron LA, Buchwald D. A review of the evidence for overlap among unexplained clinical conditions. Ann Intern Med 2001; 134:868-881.

2 Aaron LA, Buchwald D. Chronic diffuse musculoskeletal pain, fibromyalgia and co-morbid unexplained clinical conditions. Best

Pract Res Clin Rheumatol 2003; 17:563-574.

3 Hurst AF, Knott FA. Intestinal carbohydrate dyspepsia. Q J Med 1930-1931; 24:171-180.

4 Crook WG. The Yeast Connection and the woman. $3^{\text {rd }}$ ed. Jackson TN: Professional Books; 1995.

5 Eaton KK. Gut fermentation: an appraisal of an old condition with diagnostic tests and management: discussion paper. J Roy Soc Med 1991; 4:669-671.

6 Eaton KK, Chan R, Howard MA, McLaren-Howard JM. A comparison of lactulose breath hydrogen measurements with gut fermentation profiles in patients with fungal-type dysbiosis. J Nutr Environ Med 2001; 11:33-42. 
7 Eaton KK, Howard MA. Fungal-type dysbiosis of the gut: the occurrence of fungal diseases and the response to challenge with yeasty and mould containing foods. J Nutr Environ Med 1998; 8:247-255.

8 Eaton KK, Howard M, Hunnisett A. Urinary histidine excretion in patients with classical allergy (type A allergy), food intolerance (type B allergy), and fungal-type dysbiosis. J Nutr Biochem 1998; 9:586-590.

9 Eaton KK. Is there an allergic and fermentative gut condition, and does it relate to Candida? In: Brostoff J, Challacombe S (eds): Food Allergy and Intolerance. London: Saunders; 2002:351-363.

10 McLaren Howard J. Intestinal dysbiosis. Complementary Therapies in Medicine 1993; 1:153-157.

11 Eaton KK, McLaren Howard J, Hunnisett A, Harris M. Abnormal gut fermentation: laboratory studies reveal deficiency of Bvitamins, zinc and magnesium. J Nutr Biochem 1993; 4:635-638.

12 Eaton KK, Howard M, Hunnisett A. Urinary beta-alanine excretion is a marker of abnormal as well as normal gut fermentation. $\mathrm{J}$ Nutr Med 1994; 4:157-163.

13 Cormane RH, Goslings RO. Factors influencing the growth of Candida albicans. Sabouraudia 1963; 3:52-63.

14 Olsen I, Birkenland JM. Initiation and aggravation of denture stomatitis by sucrose rinses. Scand J Dent Res 1976; 84:94-97.

15 Larmas M, Makinen KK, Scheinin A. Turku sugar studies: An intermediate report on the effect of sucrose, fructose and xylitol

diets on the numbers of salivary lactobacilli, candida and streptococci. Acta Odontol Scand 1974; 32:423-433.

16 Knoke M. Gastrointestinal microecology of humans and Candida. Mycosai 1999; (suppl 1):30-34.

17 Bernhardt H, Knoke M. Mycological aspects of gastrointestinal microflora. Scand J Gastroenterol 1997; 32(suppl 222):102-106.

18 Cohen R, Roth FJ, Delgado E, Ahearn DG, Kalser MH. Fungal flora of the normal human small and large intestine. N Engl J Med 1969; 280: 638-641.

19 Giuliano M, Barza M, Jakobus NV, Gorbach SL. Effect of broad-spectrum parenteral antibiotics on composition of intestinal microflora of humans. Antimicrob Agents Chemother 1987; 31:202-206.

20 Seelig MS. Mechanisms by which antibiotics increase the incidence and severity of candidiasis and alter the immunological defences. Bacteriol Rev 1 1966; 30:4442-4459.

21 Weig M, Werner E, Frosh M, Kasper H. Limited effect of refined carbohydrate dietary supplementation on colonization of the gastrointestinal tract of healthy subjects by Candida albicans. Am J Clin Nutr 1999; 69:1170-1173.

22 Kennedy MJ, Volz PA. Ecology of Candida albicans gut colonisation: inhibition of Candida adhesion, colonisation, and dissemination from the gastrointestinal tract by bacterial antagonism. Infect Immun 1985; 49:654-663.

23 Fitzsimmons N, Berry DR. Inhibition of Candida albicans by Lactobacillus acidophilus: evidence for the involvement of a peroxidase system. Microbios 1994; 80:125-133.

24 Vartivarian SE. Virulence properties and nonimmune pathogenic mechanisms of fungi. Clin Infect Dis 1992; 14(suppl 1):30-36. 25 Shah DT, Jackman S, Engle J, Larsen B. Effect of gliotoxin on human polymorphonuclear neutrophils. Inf Dis Obster Gynecol 1998; 6:168-175.

26 Poulain D, Hopwood V, Vernes A. Antigenic variability of candida albicans. CRC Crit Rev Microbiol 1985; 12:223-70.

27 Manning M, Mitchell TG. Analysis of cytoplasmic antigens of the yeast and mycelial phases of Candida albicans by twodimensional electrophoresis. Infect Immun 1980; 30:484-495.

28 Koivikko A, et al.. Allergenic cross-reactivity of yeasts. Allergy 1988; 43:192-200.

29 Breitenbach M, Crameri R, Lehrer SB (eds): Fungal Allergy and Pathogenicity. Chem Immunol. Basel, Karger, 2002; 81:73-93.

30 Vojdani A, Rahimian P, Kalhor H and Mordechai E. Immunological cross reactivity between candida albicans and human tissue. J Clin Lab Immunol 1996; 48:1-15.

31 Nieuwenhuizen WF, Pieters RH, Knippels LM, Jansen MC, Koppelman GJ. Is Candida albicans a trigger for the onset of coeliac disease? Lancet 2003; 361:2152-2154.

32 Barclay GR, McKenzie H, Pennington J, Parratt D, Pennington CR. The effect of dietary yeast on the activity of stable chronic Crohn's disease. Scand J Gastroenterol 1992; 27:196-200.

33 Romani L, Bistoni F, Puccetti P. Initiation of T-helper cell immunity to Candida albicans by IL-12: the role of neutrophils. Chem Immunol. 1997; 68:110-35.

34 Nosal R. Histamine release from isolated rat mastcells due to glycoprotein from candida albicans in vitro. J Hyg Epidemiol Microbiol Immunol 1974; 18:377-378.

35 Kanda N, Tani K, Enomoto U, Nakai K \& Watanabe S. The skin fungus-induced Th1- and Th2-related cytokine, chemokine and prostaglandin $\mathrm{E}_{2}$ production in peripheral blood mononuclear cells from patients with atopic dermatitis and psoriasis vulgaris. Clinical \& Experimental Allergy; 32(8):1243-50.

36 Brandtzaeg P. The mucosal B cell and its functions. In: Brostoff J, Challacombe S (eds): Food Allergy and Intolerance. London: Saunders; 2002:127-171.

37 Reinholdt J, Krogh P, Holmstrup P. Degradation of IgA1, IgA2, and S-IgA by candida and torulopsis species. Acta Path Microbiol Immunol Scand, Sect C 1987; 95:65-74.

38 Levine J, Dykoski RK, Janoff EN. Candida-associated diarrhea: a syndrome in search of credibility. Clin Infect Dis 1995; 21:881886.

39 Renfro L, Feder HM Jr, Lane TJ, Manu P, Matthews DA. Yeast connection among 100 patients with chronic fatigue. Am J Med 1989; 86:165-168.

40 Petitpierre M, Gumowski P, Girard JP. Irritable bowel syndrome and hypersensitivity to food. Ann Allergy 1985; 54:538-540.

41 Nermes M, Savolainen J, Kalimo K, Lammintausta K, Viander M. Determination of IgE antibodies to Candida albicans mannan with nitrocellulose-RAST in patients with atopic diseases. Clin Exp Allergy 1994; 24:318-323.

42 Kozinn PJ, Taschdjian CL. Enteric candidasis: diagnosis and clinical considerations. Pediatrics 1962; 30:71-85.

43 Middleton SJ, Coley A, Hunter JO. The role of faecal Candida albicans in the pathogenesis of food- intolerant irritable bowel syndrome. Postgraduate Medical Journal 1992; 68:453-454.

44 Kodsi BE WP, Kozinn PJ, Iswara K, Goldberg PK. Candida esophagitis. Gastroenterology 1976; 71:715-719.

45 Jones AW, Mardh G, Anggard E. Determination of endogenous ethanol in blood and breath by gas chromatography-mass spectrometry. Pharm Biochem Behav 1983; 18(suppl):267-272.

46 Hunnisett A, Howard J, Davies D. Gut fermentation (or the 'auto-brewery') syndrome: a new clinical tests with initial observations and discussion of clinical and biochemical implications. J Nutr Med 1990; 1:33-38. 
47 Truss CO. Tissue injury induced by C. albicans: mental and neurological manifestations. J Orthomolecular Psychiatry 1978; 7:1737

48 Kroker GF. Chronic candidiasis and allergy. In: Brostoff J, Challacombe S

(eds): Food Allergy and Intolerance. London: Baillere Tindall; 1986:850-70.

49 Sehnert KW, Mathews-Larson J. Candida-related complex (CRC), a complicating factor in the treatment and diagnostic screening for alcoholics: a pilot study of 213 patients. Int J Biosoc Med Res 1991; 13:67-76.

50 Walsh TJ, Hathorn JW, Sobel JD, Merz WG, Sanchez V, Maret SM, et al.. Detection of circulating Candida enolase by

immunoassay in patients with cancer and invasive candidiasis. New Engl J Med 1991; 324:1026-1031.

51 Matthews RC, Burnie JP, Tabaqchali S. Immunoblot analysis of the serological response in systemic candidosis. Lancet 1984;

i:1415-1418.

52 Wojdani A, Ghoneum M, Cheung GP. Measurements of humoral and cellular immunity for the diagnosis of candidiasis. Clinical Ecology 1986; 3:201-207.

53 Lanson S. Immune complexes to candida mannan: An objective marker of candida overgrowth. J Adv Med 10(1997); 179-186. 54 Crandall M. The pathogenetic significance of intestinal Candida colonization. Int J Hyg Environ Health 207(2004); 79-81.

55 Santelmann H, Lærum E, Rønnevig J and Fagertun HE. Effectiveness of nystatin in polysymptomatic patients. A randomized, double-blind trial with nystatin versus placebo in general practice. Family Practice 2001; 18(3):258-265.

56 Sappington TS, McCarten WG, Hartmann CL. Diarrhoea and Candida albicans infestation of the colon. Med Ann Dist Columbia $1963 ; 32: 48-58$

57 Friedel MMK. Meteorism through yeast. Outcome of a multi-center practice-study with Ampho-Moronal. Therapiewoche 1988; 38:3706-3709.

58 Dismukes WE, Wade JS, Lee JY, Dockery BK, Hain JD. A randomised, double blind trial of nystatin therapy for the candidiasis hypersensitivity syndrome. N Engl J Med 1990; 323:1717-1723.

Appendix 1

\section{Questionnaire for Fungus-Related Disease FRDQ-7}

developed by Heiko Santelmann, MD

\begin{tabular}{|l|l|c|c|}
\hline & & NO & YES \\
\hline $\mathbf{1}$ & $\begin{array}{l}\text { Have you, at any time in your life, taken "broad spectrum" } \\
\text { antibiotics ? }\end{array}$ & $\mathbf{0}$ & $\mathbf{3}$ \\
\hline $\mathbf{2}$ & $\begin{array}{l}\text { Have you taken tetracycline or other broad spectrum } \\
\text { antibiotics for one month or longer ? }\end{array}$ & $\mathbf{0}$ & $\mathbf{3}$ \\
\hline $\mathbf{3}$ & $\begin{array}{l}\text { Are your symptoms worse on damp, muggy days or in } \\
\text { mouldy places ? }\end{array}$ & $\mathbf{0}$ & $\mathbf{3}$ \\
\hline $\mathbf{4}$ & Do you crave sugar ? & $\mathbf{0}$ & $\mathbf{3}$ \\
\hline $\mathbf{5}$ & Do you have a feeling of being "drained" ? & $\mathbf{0}$ & \\
\hline & - occasional or mild & & $\mathbf{1}$ \\
\hline & - frequent or moderately severe & & $\mathbf{2}$ \\
\hline & - severe or disabling & & $\mathbf{3}$ \\
\hline $\mathbf{6}$ & Are you bothered with vaginal burning, itching or & $\mathbf{0}$ & \\
\hline
\end{tabular}




\begin{tabular}{|l|l|l|}
\hline discharge & & \\
\hline (do you have similar symptoms from the penis) ? & & \\
\hline - occasional or mild & & $\mathbf{1}$ \\
\hline & - frequent or moderately severe & $\mathbf{2}$ \\
\hline $\mathbf{7}$ - severe or disabling & & $\mathbf{3}$ \\
\hline & $\mathbf{0}$ & \\
\hline & & $\mathbf{1}$ \\
\hline & - occasional or mild bothered by burning, itching or tearing of eyes ? & $\mathbf{2}$ \\
\hline & - frequent or moderately severe & $\mathbf{3}$ \\
\hline & - severe or disabling & \\
\hline & & \\
\hline
\end{tabular}

\author{
Score 0-3 $=$ FRD unlikely \\ Score 4-9 $=$ FRD probable \\ Score 10-21 $=$ FRD almost certain
}

Appendix 2

\title{
Test Diet \\ for FRD
}

\section{FOODS TO BE AVOIDED}

Sugar - in malt, honey, maple syrup and sweetened food, such as fizzy drinks (soda pop) and fruit cordials, ice cream, cakes, pastries, pies, biscuits, buns, chocolate, sweets and candy, chewing gum, ready-sweetened breakfast cereals, canned fruits and vegetables and sweet potatoes.

Yeast - in bread, rolls, bread-mixes, breadcrumbs, bread pudding, bread stuffing, tortillas, sourdough, beer, brewer's yeast, fermented soy sauce, pickles, cough syrup, alcoholic beverages, soy sauce, nutritional supplements containing yeast.

Mould proteins - in soy fat (-margarine and -oils), vinegar (mustard, dressing, ketchup, pickles, etc.) smoked meat, smoked fish, mushrooms, cheese, unrefrigerated orange juice, leftovers and E 330-333 (citric acid).

Milk sugar - in milk and milk products like yogurt, sour cream, milky sweets and milk sugar (=lactose) as an ingredient in prepared foods and lactose-containing tablets. 
Medications - such as antibiotics (Penicillin etc.), Prednisone, antihistamines (allergy tablets) should be discontinued latest 5 days after starting this elimination diet, in order to be able to recognise allergic reactions to foods during provocation.

NB: Also avoid food products you already know that you don't tolerate.

\section{FOODS YOU CAN EAT}

- Meat, fish, egg, chicken (but not marinated)

- All vegetables (fresh and frozen), potatoes, sprouts (if not mouldy)

- Rice, grain, flour, pasta

- Fruits (fresh and frozen), which have been washed and peeled, maximum about $1 / 2 \mathrm{lb}$. (250 g) a day. (Avoid grapes, plums and over-ripe fruit such as bananas with brown spots and soft pears, which contain lots of fructose.)

- Soda bread, crisp bread without yeast and malt, rice cakes

- Butter, margarine without soy oil

- Nuts and seeds

- Small amounts of milk as in bread, for example; cream cheese; soy milk; rice milk

- Freshly squeezed juice, (sparkling) water, mould free herb tea, a few cups of black tea and coffee, gin and vodka.

- Sugar-free popcorn, potato chips (avoid soy oil)

- Artificial sweeteners like aspartame, saccharin and xylitol.

\section{PROVOCATION TEST}

After a minimum of 9 days on this elimination diet, you will be able to test the different types of food that you have avoided by reintroducing food in the following manner:

- 1st day: $\quad \frac{1 / 4}{4}$ teaspoon vinegar in a glass of water or in a salad

- 2nd day: $\quad$ yeast from beer ( $1 / 4$ glass) or 1 brewer's yeast tablet

- 3rd day: $\quad 1$ pint milk or sour cream

- 4th day: $\quad$ ordinary bread (with yeast)

- 5th day: $\quad$ sugar, honey or chocolate

After this you can test other food that you wonder about such as wine, sweeteners, canned food and so on.

Do the test preferably at breakfast. If you have a reaction, such as an itch, you have to postpone the testing of other foods until the reaction is gone.

If you react to the food on the 1st, 2nd, or 4th day within 24 hours, it probably indicates a cross allergy to yeast antigens. If you react to milk, you might have milk sugar (lactose) intolerance.

If you are not quite sure if you reacted to a product and want to test it again, you have to wait at least 4 days (washout-phase) before doing so.

Sugar should be tested last because the reactions may be delayed for 2-3 days.

Food products that you have had a reaction to must be avoided for at least 2 months ( 3 months if you react to moulds / vinegar) while you are taking an effective antifungal medication and probiotic (Acidophilus and Bifidobacter), before you try them again.

If you have a high score on the FRDQ-7 and did not experience a significant improvement on this diet within 2 weeks, you should not start a treatment with this diet and antifungals, but seek the help of a qualified health practitioner because there may be associated causes such as a chemical sensitivity, mercury allergy or a food or gluten intolerance. Additional tests should be performed such as a test for gluten- and gliadin-IgA. 